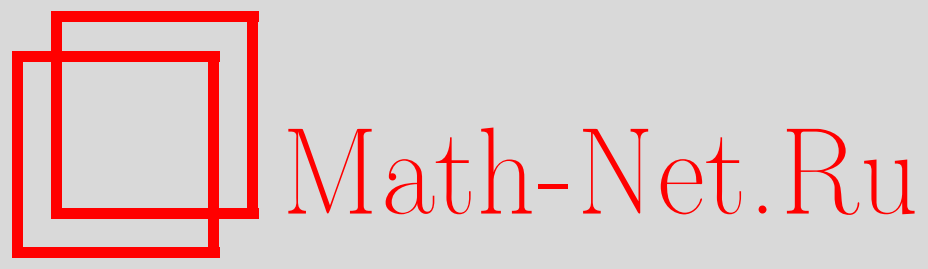

Б. А. Дубровин, С. А. Зыков, М. В. Павлов, Слабо нелинейные гамильтоновы уравнения в частных производных и новый класс решений уравнений ассоциативности WDVV, Функи. анализ и его прил., 2011, том 45, выпуск 4, 49-64

DOI: https://doi.org/10.4213/faa3053

Использование Общероссийского математического портала MathNet.Ru подразумевает, что вы прочитали и согласны с пользовательским соглашением

http://www . mathnet.ru/rus/agreement

Параметры загрузки:

IP : 18.234 .197 .8

26 апреля 2023 г., 15:46:15

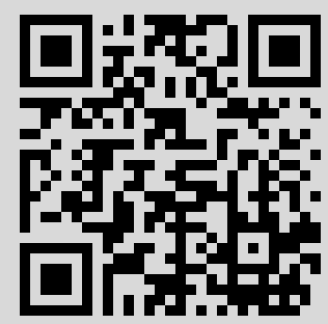




\title{
Слабо нелинейные гамильтоновы уравнения в частных производных и новый класс решений уравнений ассоциативности WDVV*
}

\author{
(c) 2011. Б. А. Дуьровин, С. А. Зыков, М. В. ПАвлов
}

Памяти Владимира Игоревича Арнольда

\begin{abstract}
Мы вводим новый класс решений уравнений ассоциативности WDVV. Этот класс выделен свойством, что одно из коммутирующих уравнений в частных производных, связанных с решением уравнений WDVV, является слабо нелинейным. Мы сводим проблему классификации таких решений уравнений WDVV к частному случаю так называемого алгебраического матричного уравнения Риккати, получая полную классификацию неприводимых решений.
\end{abstract}

\section{§1. Введение}

Уравнения ассоциативности Е. Виттена-Р. Дийкграафа-Е. Верлинде-Х. Верлинде (WDVV) - это переопределенная система уравнений в частных производных

$\frac{\partial^{3} F}{\partial v^{\alpha} \partial v^{\beta} \partial v^{\lambda}} \eta^{\lambda \mu} \frac{\partial^{3} F}{\partial v^{\mu} \partial v^{\gamma} \partial v^{\delta}}=\frac{\partial^{3} F}{\partial v^{\delta} \partial v^{\beta} \partial v^{\lambda}} \eta^{\lambda \mu} \frac{\partial^{3} F}{\partial v^{\mu} \partial v^{\gamma} \partial v^{\alpha}}, \quad \alpha, \beta, \gamma, \delta=1, \ldots, n$,

для функции $F=F(\mathbf{v}), \mathbf{v}=\left(v^{1}, \ldots, v^{n}\right)$, удовлетворяющей условию

$$
\frac{\partial^{3} F}{\partial v^{\alpha} \partial v^{\beta} \partial v^{1}}=\eta_{\alpha \beta} \text {. }
$$

Здесь $\left(\eta_{\alpha \beta}\right)_{1 \leqslant \alpha, \beta \leqslant n}$ и $\left(\eta^{\alpha \beta}\right)_{1 \leqslant \alpha, \beta \leqslant n}-$ постоянные симметрические невырожденные взаимно обратные матрицы, $\eta_{\alpha \lambda} \eta^{\lambda \beta}=\delta_{\alpha}^{\beta}$. Везде в этом параграфе подразумевается суммирование по повторяющимся индексам, обозначенным греческими буквами.

Напомним [5], что существует взаимно однозначное соответствие между решениями уравнений ассоциативности WDVV и $n$-параметрическими семействами $n$-мерных коммутативных ассоциативных алгебр

$$
\mathscr{A}_{\mathbf{v}}=\operatorname{span}\left(e_{1}, \ldots, e_{n}\right)
$$

*Работа частично поддержана European Research Council Advanced Grant FroM-PDE, грантом Правительства Российской Федерации 2010-220-01-077 (договор №11.G34.31.0005), а также грантом «Геометрические методы в теории нелинейных волн и их приложения» PRIN 2008 Министерства университетов и исследований Италии. Исследования третьего из авторов частично поддержаны грантом Президиума РАН «Фундаментальные проблемы в нелинейной динамике», а также грантом РФФИ 11-01-00197. Второй и третий авторы выражают благодарность институту SISSA в Триесте (Италия), где была сделана часть этой работы. Второй автор также выражает благодарность отделению INFN в г. Лечче за поддержку по проекту LE41. 
с единицей $e=e_{1}$, оснащенных симметрической невырожденной инвариантной билинейной формой $(, \quad)$, такой, что структурные константы выражаются через третьи производные функции $F$, называемой потенииалом:

$$
\begin{aligned}
e_{\alpha} \cdot e_{\beta} & =c_{\alpha \beta}^{\gamma}(\mathbf{v}) e_{\gamma}, \quad \alpha, \beta=1, \ldots, n, \\
e_{1} \cdot e_{\alpha} & =e_{\alpha} \quad \text { для всех } \alpha, \\
\left(e_{\alpha}, e_{\beta}\right) & =\eta_{\alpha \beta}, \\
\left(e_{\alpha} \cdot e_{\beta}, e_{\gamma}\right) & =\left(e_{\alpha}, e_{\beta} \cdot e_{\gamma}\right)=\eta_{\gamma \lambda} c_{\alpha \beta}^{\lambda}(\mathbf{v})=\frac{\partial^{3} F(\mathbf{v})}{\partial v^{\alpha} \partial v^{\beta} \partial v^{\gamma}} .
\end{aligned}
$$

Если, кроме того, функция $F$ удовлетворяет определенному условию квазиоднородности, то мы получаем локальное описание фробениусовых многообразий (подробнее см. в [5]). На этих многообразиях определена естественная метрика

$$
d s^{2}=\eta_{\alpha \beta} d v^{\alpha} d v^{\beta}
$$

(не обязательно положительно определенная). Переменные $v^{1}, \ldots, v^{n}$ являются плоскими координатами для этой метрики. Алгебра $\mathscr{A}_{\mathbf{v}}$ отождествляется с касательным пространством к многообразию в точке $\mathbf{v}$,

$$
e_{\alpha} \leftrightarrow \frac{\partial}{\partial v^{\alpha}}
$$

Более подробно о бескоординатном геометрическом описании фробениусовых многообразий см. в [5].

Решение уравнений ассоциативности (1.1) называется полупростым, если алгебра $\mathscr{A}_{\mathbf{v}}$ не имеет нильпотентных элементов в общей точке $\mathbf{v}$. В полупростом случае было доказано [4] существование локальных канонических координат $u_{i}=u_{i}(\mathbf{v}), i=1, \ldots, n$, таких, что таблица умножения принимает следующую стандартную форму:

$$
\frac{\partial}{\partial u_{i}} \cdot \frac{\partial}{\partial u_{j}}=\delta_{i j} \frac{\partial}{\partial u_{i}} .
$$

В этих канонических координатах метрика (1.2) становится диагональной:

$$
d s^{2}=\sum_{i=1}^{n} h_{i}^{2}(\mathbf{u}) d u_{i}^{2} .
$$

Более того, это егоровская метрика (см. [7]), т. е. коэфбиииенты вращения

$$
\gamma_{i j}(\mathbf{u})=\frac{1}{h_{j}} \frac{\partial h_{i}}{\partial u_{j}}
$$

являются симметричными по $i, j, \gamma_{j i}=\gamma_{i j}$. Коэффициенты вращения $\gamma_{i j}$ удовлетворяют следующей системе уравнений Дарбу-Егорова [1]:

$$
\begin{array}{rlrl}
\frac{\partial \gamma_{i j}}{\partial u_{k}} & =\gamma_{i k} \gamma_{k j}, & & i, j, k \text { различны, } \\
\sum_{k=1}^{n} \frac{\partial \gamma_{i j}}{\partial u_{k}} & =0, & i \neq j .
\end{array}
$$


Любое решение системы уравнений Дарбу-Егорова получается из полупростого решения уравнений ассоциативности WDVV. Процедура восстановления последнего опирается на решения следующей системы линейных дифференциальных уравнений на вектор-функцию $\psi=\left(\psi_{1}(\mathbf{u}), \ldots, \psi_{n}(\mathbf{u})\right)$ :

$$
\begin{aligned}
\frac{\partial \psi_{i}}{\partial u_{j}} & =\gamma_{i j} \psi_{j}, \quad i \neq j, \\
\sum_{k=1}^{n} \frac{\partial \psi}{\partial u_{k}} & =0 .
\end{aligned}
$$

Обозначим через $\psi_{i \alpha}=\psi_{i \alpha}(\mathbf{u}), \alpha=1, \ldots, n$, систему $n$ линейно независимых решений системы (1.6)-(1.7). Реконструкция зависит от выбора одного из этих решений в качестве коэффициентов Ламе инвариантой метрики (1.2); положим $\alpha=1, h_{i}=\psi_{i 1}$. Тогда

$$
\begin{aligned}
\eta_{\alpha \beta} & =\sum_{i=1}^{n} \psi_{i \alpha} \psi_{i \beta}, \\
d v_{\alpha} & =\sum_{i=1}^{n} \psi_{i \alpha} \psi_{i 1} d u_{i}, \\
\frac{\partial^{3} F}{\partial v^{\alpha} \partial v^{\beta} \partial v^{\gamma}} & =\sum_{i=1}^{n} \frac{\psi_{i \alpha} \psi_{i \beta} \psi_{i \gamma}}{\psi_{i 1}} .
\end{aligned}
$$

Отметим также следующую формулу для дифференциалов вторых производных

$$
\Omega_{\alpha \beta}=\frac{\partial^{2} F}{\partial v^{\alpha} \partial v^{\beta}}
$$

потенциала $F$ :

$$
d \Omega_{\alpha \beta}=\sum_{i=1}^{n} \psi_{i \alpha} \psi_{i \beta} d u_{i} .
$$

Как было показано в [3], система Дарбу-Егорова (1.4)-(1.5) может быть отождествлена со специальной редукцией хорошо известной в теории интегрируемых уравнений в частных производных системы $n$-волн, записанной в форме, предложенной в [2]. Она может быть вложена в схему системы $n \mathrm{~K}$ (см., например, [10]). Все известные частные решения уравнений ассоциативности соответствуют дальнейшей редукции системы $n$-волн в систему обыкновенных дифференциальных уравнений. Например, полупростые фробениусовы многообразия выделяются добавлением условия однородности коэффициентов вращения

$$
\sum_{k=1}^{n} u_{k} \frac{\partial \gamma_{i j}}{\partial u_{k}}=-\gamma_{i j}, \quad i \neq j .
$$

Это условие соответствует аксиоме квазиоднородности теории фробениусовых многообразий ([4], [5]). Другие частные классы решений (солитоны, алгеброгеометрические решения, вырожденные фробениусовы многообразия) также естественным образом возникают в схеме системы $n$-волн. 
В этой работе мы вводим другой класс решений уравнений WDVV. Для описания этого класса сначала напомним связь между уравнениями ассоциативности и интегрируемыми иерархиями. Пусть $\theta=\theta(\mathbf{v})-$ решение следующей системы линейных дифференциальных уравнений:

$$
\frac{\partial^{2} \theta}{\partial v^{\alpha} \partial v^{\beta}}=c_{\alpha \beta}^{\gamma} \frac{\partial^{2} \theta}{\partial v^{1} \partial v^{\gamma}}, \quad \alpha, \beta=1, \ldots, n .
$$

Рассмотрим систему квазилинейных уравнений в частных производных первого порядка на вектор-функцию $\mathbf{v}=\mathbf{v}(x, t)$

$$
\mathbf{v}_{t}=[\nabla \theta(\mathbf{v})]_{x} .
$$

Это гамильтоновы уравнения в частных производных первого порядка с гамильтонианом $H=\int \theta(\mathbf{v}) d x$ и скобкой Пуассона $\left\{v^{\alpha}(x), v^{\beta}(y)\right\}=\eta^{\alpha \beta} \delta^{\prime}(x-y)$ (см. [6]). Все эти гамильтоновы системы вида (1.10), (1.11) попарно коммутируют. Более того, гамильтонианы (1.10) удовлетворяют определенным условиям полноты (см. [11]). Итак, любую из этих систем (1.11) можно рассматривать как вполне интегрируемую гамильтонову систему уравнений в частных производных.

В полупростом случае все такие уравнения в частных производных диагонализуются в канонических координатах:

$$
\mathbf{u}_{t}=\Lambda(\mathbf{u}) \mathbf{u}_{x}, \quad \Lambda(\mathbf{u})=\operatorname{diag}\left(\lambda_{1}(\mathbf{u}), \ldots, \lambda_{n}(\mathbf{u})\right) .
$$

Таким образом, канонические координаты являются инвариантами Римана для квазилинейных систем (1.11). Для общего решения системы (1.10) характеристические скорости в общей точке $\mathbf{u}$ попарно различны,

$$
\lambda_{i}(\mathbf{u}) \neq \lambda_{j}(\mathbf{u}), \quad i \neq j .
$$

Определение 1.1. Полупростое решение $F(\mathbf{v})$ уравнений ассоциативности WDVV называется слабо нелинейным, если среди коммутирующих уравнений в частных производных (1.10)-(1.12) существует по крайней мере одно, удовлетворяющее условию (1.13), а также условию

$$
\frac{\partial \lambda_{i}(\mathbf{u})}{\partial u_{i}}=0, \quad i=1, \ldots, n .
$$

Наша терминология основана на том, что одна из квазилинейных коммутирующих систем (1.10)-(1.12) является слабо нелинейной, т. е. $i$-я характеристическая скорость $\lambda_{i}$ не зависит от $i$-го инварианта Римана $u_{i}$ для каждого $i=1, \ldots, n$.

Основная цель настоящей работы - классифицировать слабо нелинейные решения уравнений ассоциативности WDVV. Такое решение называется приво$\partial u м ы м$, если $\gamma_{i j}(\mathbf{u}) \equiv 0$ для некоторого $i$ при любом $j \neq i$. В противном случае такое решение будем называть неприводимым. Достаточно классифицировать неприводимые слабо нелинейные решения.

Теорема 1.2. Коэфбициенты вращения неприводимого слабо нелинейного решения уравнений ассоциативности $W D V V$ имеют вид

$$
\gamma_{i j}(u)=\frac{\left[G\left(1-\frac{1}{\rho} \operatorname{th} \rho U \cdot G\right)^{-1}\right]_{i j}}{\operatorname{ch} \rho u_{i} \operatorname{ch} \rho u_{j}}, \quad i, j=1, \ldots, n, i \neq j,
$$


где $U=\operatorname{diag}\left(u_{1}, \ldots, u_{n}\right)$, a $G$ - симметрическая матрица, удовлетворяющая условию $G^{2}=\rho^{2} \cdot 1, \rho-$ произвольный комплексный параметр.

При $\rho=0$ формулы (1.14) сохраняют смысл, если принять во внимание известные пределы

$$
\frac{1}{\rho} \operatorname{th} \rho U \rightarrow U, \quad \operatorname{ch} \rho u_{i} \rightarrow 1 .
$$

Работа организована следующим образом. В $\S 2$ мы напоминаем необходимые конструкции из теории уравнений ассоциативности WDVV. Мы выводим основную систему дифференциальных уравнений (2.6) теории слабо нелинейных решений WDVV. В $\S 3$ мы решаем эту основную систему и описываем ее группу симметрий, действующих с помощью дробно-линейных преобразований. В $\S 4$ мы отбираем те решения основной системы, которые приводят к уравнениям WDVV, и выводим матричное алгебраическое уравнение Риккати. Используя симметрии этого уравнения, мы классифицируем все неприводимые слабо нелинейные решения уравнений ассоциативности WDVV.

Благодарности. Авторы благодарят Е. В. Ферапонтова и С. П. Царева за стимулирующие и плодотворные обсуждения.

\section{§2. Слабо нелинейные решения уравнений ассоциативности WDVV}

Пусть $\Gamma=\left(\gamma_{i j}(\mathbf{u})\right)_{1 \leqslant i, j \leqslant n}-$ симметрическая матрица коэффициентов вращения $^{1)}$ (1.3) слабо нелинейного неприводимого решения уравнений ассоциативности WDVV.

Лемма 2.1. Матричнозначная функиия $\Gamma=\Gamma(\mathbf{u})$ удовлетворяет следующей системе дифференциальных уравнений:

$$
\frac{\partial \Gamma}{\partial u_{k}}=\Gamma E_{k} \Gamma+\sigma_{k}\left(u_{k}\right) E_{k}, \quad k=1, \ldots, n,
$$

где $\sigma_{1}\left(u_{1}\right), \ldots, \sigma_{n}\left(u_{n}\right)$ - некоторье функции. Здесь $E_{k}-$ матрица с единственным ненулевым элементом,

$$
\left(E_{k}\right)_{i j}=\delta_{i k} \delta_{j k}
$$

Доказательство. По определению уравнения

$$
\frac{\partial \gamma_{i j}}{\partial u_{k}}=\gamma_{i k} \gamma_{k j}
$$

выполняются, когда индексы $i, j, k$ различны. Сначала установим справедливость (2.3) также для $k=i$ или $k=j$ с $i \neq j$ или для $i=j$, но $k \neq i$.

Согласно [4], характеристические скорости $\lambda_{k}(\mathbf{u})$ коммутирующих уравнений в частных производных (1.10)-(1.12) могут быть представлены в форме

$$
\lambda_{k}(\mathbf{u})=\frac{\phi_{k}(\mathbf{u})}{h_{k}(\mathbf{u})}, \quad k=1, \ldots, n,
$$

1) На самом деле в дифференциальной геометрии криволинейных ортогональных систем координат только внедиагональные элементы матрицы $\Gamma$ называются коэффициентами вращения. Однако в нашем случае будет удобно также добавить диагональные элементы $\gamma_{i i}=\partial \log h_{i} / \partial u_{i}$. 
где вектор-функция $\phi=\left(\phi_{1}(\mathbf{u}), \ldots, \phi_{n}(\mathbf{u})\right)$ удовлетворяет системе линейных дифференциальных уравнений

$$
\frac{\partial \phi_{i}}{\partial u_{j}}=\gamma_{i j} \phi_{j}, \quad i \neq j .
$$

$\mathrm{B}$ частности, $\phi_{k}=h_{k}$ - одно из решений системы (2.4). Пусть $\phi-$ одно из решений системы (2.4), соответствующее слабо нелинейному элементу семейства коммутирующих потоков (1.10)-(1.12). Дифференцируя уравнение

$$
\frac{\partial}{\partial u_{k}}\left(\frac{\phi_{k}}{h_{k}}\right)=0
$$

по $u_{i}$ с $i \neq k$, получаем следующее уравнение:

$$
\frac{h_{i}}{h_{k}}\left(\lambda_{i}-\lambda_{k}\right) \gamma_{i k} \frac{\partial}{\partial u_{k}}\left[\log \gamma_{i k}-\log h_{k}\right]=0 .
$$

В силу неприводимости и свойства (1.13) из него следует, что

$$
\frac{\partial \log \gamma_{i k}}{\partial u_{k}}=\frac{\partial \log h_{k}}{\partial u_{k}}=\gamma_{k k} .
$$

Это доказывает (2.3) для $k=j, i \neq j$. Затем при $k \neq i$ получаем

$$
\frac{\partial \gamma_{i i}}{\partial u_{k}}=\frac{\partial}{\partial u_{i}} \frac{\partial \log h_{i}}{\partial u_{k}}=\frac{\partial}{\partial u_{i}}\left(\gamma_{i k} \frac{h_{k}}{h_{i}}\right)=\gamma_{i k}^{2} .
$$

Таким образом, уравнение (2.3) при $i=j$ и $k \neq i$ также проверено. Последний шаг - необходимо доказать, что разность $\sigma_{i}:=\partial \gamma_{i i} / \partial u_{i}-\gamma_{i i}^{2}$ зависит только от $u_{i}$. В самом деле, для $k \neq i$

$$
\frac{\partial}{\partial u_{k}}\left(\frac{\partial \gamma_{i i}}{\partial u_{i}}-\gamma_{i i}^{2}\right)=\frac{\partial}{\partial u_{i}} \frac{\partial \gamma_{i i}}{\partial u_{k}}-2 \gamma_{i i} \gamma_{i k}^{2}=\frac{\partial \gamma_{i k}^{2}}{\partial u_{i}}-2 \gamma_{i i} \gamma_{i k}^{2}=0 .
$$

Теперь опишем класс преобразований

$$
u_{k} \mapsto \tilde{u}_{k}, \quad \gamma_{i j} \mapsto \tilde{\gamma}_{i j},
$$

оставляющих систему (2.1) инвариантной.

Лемма 2.2. Подстановка

$$
\begin{array}{ll}
\tilde{u}_{k}=f_{k}\left(u_{k}\right), & k=1, \ldots, n, \\
\tilde{\gamma}_{i j}=\frac{\gamma_{i j}}{\sqrt{f_{i}^{\prime}\left(u_{i}\right) f_{j}^{\prime}\left(u_{j}\right)}}-\frac{f_{i}^{\prime \prime}\left(u_{i}\right)}{2\left[f_{i}^{\prime}\left(u_{i}\right)\right]^{2}} \delta_{i j}, & i, j=1, \ldots, n,
\end{array}
$$

с прочзвольными непостоянными гладкими функииями $f_{1}\left(u_{1}\right), \ldots, f_{n}\left(u_{n}\right)$ сохраняет вид уравнений (2.1),

$$
\frac{\partial \widetilde{\Gamma}}{\partial \tilde{u}_{k}}=\widetilde{\Gamma} E_{k} \widetilde{\Gamma}+\tilde{\sigma}_{k}\left(\tilde{u}_{k}\right) E_{k}, \quad k=1, \ldots, n,
$$

c $f_{k}^{\prime 2} \tilde{\sigma}_{k}=\sigma_{k}-\frac{1}{2} S_{u_{k}}\left(f_{k}\right)$. Здесь $S_{u}(f)-$ производная Швариа функции $f=f(u)$,

$$
S_{u}(f)=\frac{f^{\prime \prime \prime}}{f^{\prime}}-\frac{3}{2} \frac{f^{\prime \prime 2}}{f^{\prime 2}} .
$$

Доказательство получается прямым вычислением. 
Следствие 2.3. Подходящим преобразованием типа (2.5) система (2.1) моэсет быть приведена $к$ виду

$$
\frac{\partial \widetilde{\Gamma}}{\partial \tilde{u}_{k}}=\widetilde{\Gamma} E_{k} \widetilde{\Gamma}, \quad k=1, \ldots, n .
$$

Доказательство. Искомое преобразование $\tilde{u}_{k}=f_{k}\left(u_{k}\right)$ определяется из уравнений Шварца

$$
S_{u_{k}}\left(f_{k}\right)=2 \sigma_{k}\left(u_{k}\right), \quad k=1, \ldots, n \text {. }
$$

Напомним, что решение общего уравнения Шварца $S_{u}(f(u))=2 \sigma(u)$ представляется как отношение двух решений линейного уравнения второго порядка

$$
y^{\prime \prime}+\sigma(u) y=0 .
$$

Замечание 2.4. Система (2.6) изучалась в [8] при исследовании так называемой многопотоковой редукции холодного газа нелокального кинетического уравнения, выведенного из термодинамического предела усредненных многофазных решений КдФ методом Уизема.

В следующем параграфе мы решим систему (2.6).

\section{§3. Основная система}

В этом параграфе мы опишем решения основной системы

$$
\frac{\partial \Gamma}{\partial u_{k}}=\Gamma E_{k} \Gamma, \quad k=1, \ldots, n .
$$

Здесь

$$
\Gamma=\left(\gamma_{i j}(\mathbf{u})\right)_{1 \leqslant i, j \leqslant n}
$$

- симметрическая матрица (знаки «тильда», введенные в предыдущем параграфе, здесь опущены). Можно легко проверить условия совместности

$$
\frac{\partial}{\partial u_{l}} \frac{\partial \Gamma}{\partial u_{k}}=\frac{\partial}{\partial u_{k}} \frac{\partial \Gamma}{\partial u_{l}}
$$

для всех $k, l$. Итак, локально любое решение системы (3.1) однозначно определяется начальными данными

$$
\Gamma^{0}=\Gamma\left(\mathbf{u}^{0}\right)
$$

Здесь $\mathbf{u}^{0}$ - произвольная точка в пространстве независимых переменных. Таким образом, пространство решений системы $(3.1)$ имеет размерность $n(n+1) / 2$.

Без потери общности положим $\mathbf{u}^{0}=0$. Решение системы (3.1) с заданными начальными условиями в точке $\mathbf{u}=0$ может быть выписано явно.

Предложение 3.1. Решение $\Gamma=\Gamma(\mathbf{u})$ основной системъ (3.1) с началънъми данными

$$
\Gamma(0)=G
$$

әде $G=\left(g_{i j}\right)$ - заданная симметрическая матрица, определяется следующей формулой:

$$
\Gamma=G(1-U G)^{-1}
$$

где 1 - это единичная $n \times n$-матрица и $U=\operatorname{diag}\left(u_{1}, \ldots, u_{n}\right)$. 
Доказательство. Симметричность матрицы (3.2) эквивалентна равенству

$$
G(1-U G)^{-1}=(1-G U)^{-1} G .
$$

Чтобы доказать последнее, мы умножим его на $1-G U$ слева и на $1-U G$ справа. Получаем очевидное тождество $(1-G U) G=G(1-U G)=G-G U G$. Ясно, что $\Gamma(0)=G$. Применяя хорошо известное правило дифференцирования обратной матрицы

$\frac{\partial \Gamma}{\partial u_{k}}=-G(1-U G)^{-1} \frac{\partial(1-U G)}{\partial u_{k}}(1-U G)^{-1}=G(1-U G)^{-1} E_{k} G(1-U G)^{-1}=\Gamma E_{k} \Gamma$, приходим к доказательству предложения.

Пример 3.2. Для матрицы $G$ ранга $1, g_{i j}=\omega_{i} \omega_{j}$, получается следующее решение основной системы:

$$
\gamma_{i j}=\frac{\omega_{i} \omega_{j}}{1-\sum_{k=1}^{n} \omega_{k}^{2} u_{k}} .
$$

Опишем теперь подкласс преобразований (2.5), оставляющей инвариантной основную систему (3.1).

Предложение 3.3. Основная система (3.1) инвариантна относительно преобразований (2.5) тогда и только тогда, когда $f_{k}\left(u_{k}\right)$ является дробнолинейным преобразованием для каждого $k=1, \ldots, n$,

$$
f_{k}\left(u_{k}\right)=\frac{a_{k} u_{k}+b_{k}}{c_{k} u_{k}+d_{k}}, \quad a_{k} d_{k}-b_{k} c_{k}=1 .
$$

Доказательство. Хорошо известно, что общее решение однородного уравнения Шварца

$$
\frac{f^{\prime \prime \prime}}{f^{\prime}}-\frac{3}{2} \frac{f^{\prime \prime 2}}{f^{\prime 2}}=0
$$

задается дробно-линейной функцией.

Следствие 3.4. Основная система (3.1) инвариантна относительно преобразований

$$
\begin{aligned}
& \tilde{u}_{k}=\frac{a_{k} u_{k}+b_{k}}{c_{k} u_{k}+d_{k}}, \quad\left(\begin{array}{ll}
a_{k} & b_{k} \\
c_{k} & d_{k}
\end{array}\right) \in S L_{2}(\mathbb{R}), k=1, \ldots, n, \\
& \tilde{\gamma}_{i j}=\left(c_{i} u_{i}+d_{i}\right)\left(c_{j} u_{j}+d_{j}\right) \gamma_{i j}+c_{i}\left(c_{i} u_{i}+d_{i}\right) \delta_{i j}, \quad i, j=1, \ldots, n .
\end{aligned}
$$

Укажем матричную версию преобразования (3.4):

$$
\widetilde{U}=(A U+B)(C U+D)^{-1}, \quad \widetilde{\Gamma}=(C U+D) \Gamma(C U+D)+C(C U+D)
$$

где $A=\operatorname{diag}\left(a_{1}, \ldots, a_{n}\right), B=\operatorname{diag}\left(b_{1}, \ldots, b_{n}\right), C=\operatorname{diag}\left(c_{1}, \ldots, c_{n}\right), D=\operatorname{diag}\left(d_{1}\right.$, $\left.\ldots, d_{n}\right), A D-B C=1$.

Пример 3.5. Подстановка

$$
\tilde{u}_{k}=\omega_{k}^{2} u_{k}, \quad \tilde{\gamma}_{i j}=\frac{\gamma_{i j}}{\omega_{i} \omega_{j}}
$$

приводит решение (3.3) к стандартной форме

$$
\tilde{\gamma}_{i j}=\frac{1}{1-\sum_{k=1}^{n} \tilde{u}_{k}}, \quad i, j=1, \ldots, n .
$$


Действие преобразований (3.4) на решениях (3.2) задается следующим аналогом модулярных преобразований Зигеля.

Предложение 3.6. Рассмотрим симметрическую матрицу $G$, удовлетворяюшую условию $\operatorname{det}(A+B G) \neq 0$. Тогда преобразование (3.4) отображает решение $\Gamma(\mathbf{u})$ с начальными данными $\Gamma(0)=G$ в

$$
\widetilde{\Gamma}=\widetilde{G}(1-\widetilde{U} \widetilde{G})^{-1}
$$

əəe

$$
\widetilde{G}=(C+D G)(A+B G)^{-1}
$$

Доказательство. Согласно (3.5),

$$
\widetilde{\Gamma}=(-C \widetilde{U}+A)^{-1} G[A+B G-\widetilde{U}(C+D G)]^{-1}+C(-C \widetilde{U}+A)^{-1}
$$

Вычисляя начальные данные этого решения при $\tilde{\mathbf{u}}=0$, мы получаем $\widetilde{\Gamma}(0)=\widetilde{G}$, где матрица $\widetilde{G}$ задана формулой (3.6).

Определение 3.7. Два решения $Г$ и $\widetilde{\Gamma}$ основной системы называются эквивалентными, если они связаны преобразованием симметрии (3.5). Две симметрические матрицы $G$ и $\widetilde{G}$, связанные преобразованием (3.6), также будем называть эквивалентными.

Отметим полезное тождество

$$
(C+D G)(A+B G)^{-1}=(A+G B)^{-1}(C+G D),
$$

эквивалентное симметричности матрицы $G$.

\section{§4. Переход от решений основной системы к слабо нелинейным решениям уравнений ассоциативности WDVV}

В этом параграфе мы рассмотрим проблему отбора тех решений основной системы (3.1), которые получаются из слабо нелинейных решений уравнений ассоциативности WDVV.

Для данной симметрической матричнозначной функции $\Gamma(\mathbf{u})$, удовлетворяющей (3.1), мы ищем подстановку в форме (2.5), такую, что преобразованная матрица $\widetilde{\Gamma}$ удовлетворяет также последнему уравнению (1.5) системы ДарбуЕгорова, т. е.

$$
\sum_{k=1}^{n} \frac{\partial \widetilde{\Gamma}}{\partial \tilde{u}_{k}} \text { - диагональная матрица. }
$$

Напомним, что справедливость уравнений (1.4)

$$
\frac{\partial \tilde{\gamma}_{i j}}{\partial \tilde{u}_{k}}=\tilde{\gamma}_{i k} \tilde{\gamma}_{k j}, \quad \text { когда } i, j, k \text { различны, }
$$

следует из основной системы в силу леммы 2.2 .

Применяя лемму 2.2, мы приходим к следующему простому заключению.

Предложение 4.1. Пусть $Г(\mathbf{u})$ - решение основной системы (3.1). Предположим, что функиии $f_{1}\left(u_{1}\right), \ldots, f_{n}\left(u_{n}\right)$ выбраны таким образом, что преобразованная матрица $\widetilde{\Gamma}=\left(\tilde{\gamma}_{i j}\right)$ (см. (2.5)) удовлетворяет условию (4.1). Тогда внедиагональные элементы преобразованной матрицы $\widetilde{\Gamma}$ являются коэфбициентами вращения некоторой егоровской метрики. 
Введем диагональные матрицы

$$
\begin{aligned}
S=\operatorname{diag}\left(s_{1}, \ldots, s_{n}\right), & s_{i}=\frac{1}{f_{i}^{\prime}}, \\
S^{\prime}=\operatorname{diag}\left(s_{1}^{\prime}, \ldots, s_{n}^{\prime}\right), & s_{i}^{\prime}=\frac{d s_{i}}{d u_{i}}=-\frac{f_{i}^{\prime \prime}}{\left[f_{i}^{\prime}\right]^{2}} .
\end{aligned}
$$

Здесь и далее мы будем использовать сокращенные обозначения

$$
f_{i}^{\prime}=f_{i}^{\prime}\left(u_{i}\right), \quad f_{i}^{\prime \prime}=f_{i}^{\prime \prime}\left(u_{i}\right) \quad \text { и т. д. }
$$

В этих обозначениях закон преобразования (2.5) имеет вид

$$
\widetilde{\Gamma}=S^{1 / 2} \Gamma S^{1 / 2}+\frac{1}{2} S^{\prime} .
$$

Таким образом, условие (4.1) может быть записано в форме

$$
\Gamma S \Gamma+\frac{1}{2} S^{\prime} \Gamma+\frac{1}{2} \Gamma S^{\prime}+P=0
$$

для некоторой диагональной матрицы $P$.

Определение 4.2. Решение Г называется приводимым, если для некотоpыx $i$

$$
\gamma_{i j} \equiv 0 \quad \text { при } j \neq i \text {. }
$$

В противном случае оно называется неприводимым.

Приводимое решение выражается через функции, зависящие от меньшего числа переменных.

Теорема 4.3. Для неприводимого решения

$$
\Gamma=G(1-U G)^{-1}=(1-G U)^{-1} G
$$

преобразование (2.5), удовлетворлющее условию (4.1), существует тогда и только тогда, когда матрица $G$ удовлетворяет квадратному уравнению

$$
G R G+Q G+G Q+P=0
$$

для некоторых постоянных диагональных матрии,

$$
P=\operatorname{diag}\left(p_{1}, \ldots, p_{n}\right), \quad Q=\operatorname{diag}\left(q_{1}, \ldots, q_{n}\right), \quad R=\operatorname{diag}\left(r_{1}, \ldots, r_{n}\right) .
$$

Рассматриваемое преобразование имеет вид

$$
\frac{d \tilde{u}_{i}}{d u_{i}}=\frac{1}{p_{i} u_{i}^{2}+2 q_{i} u_{i}+r_{i}}, \quad i=1, \ldots, n .
$$

Доказательство. Дифференцируя (4.4) по $u_{i}$ и используя (3.1) и очевидные формулы

и т. д., получаем

$$
\frac{\partial S}{\partial u_{i}}=s_{i}^{\prime} E_{i}, \quad \frac{\partial S^{\prime}}{\partial u_{i}}=s_{i}^{\prime \prime} E_{i}
$$

$$
\left(\frac{1}{2} s_{i}^{\prime \prime}-p_{i}\right)\left(\Gamma E_{i}+E_{i} \Gamma\right)+\frac{\partial P}{\partial u_{i}}=0
$$

Все элементы матрицы $\Gamma E_{i}+E_{i} \Gamma$ обращаются в нуль за исключением $i$-й строки и $i$-го столбца, совпадающих с $\left(\gamma_{1 i}, \ldots, \gamma_{n i}\right)$. Благодаря предположению о неприводимости из (4.6) следует, что

$$
p_{i}=\frac{1}{2} s_{i}^{\prime \prime} .
$$


Подстановка в (4.6) дает

$$
\frac{\partial P}{\partial u_{i}}=0 .
$$

Повторение этой процедуры для каждого $i=1, \ldots, n$ доказывает, что матрица $P$ постоянна. Используя (4.7), приходим к заключению, что $s_{i}=s_{i}\left(u_{i}\right)$ - квадратичный полином, $s_{i}=p_{i} u_{i}^{2}+2 q_{i} u_{i}+r_{i}$. Наконец, умножая уравнение $(4.4)$ слева на $1-G U$ и справа на $1-U G$, получаем квадратное уравнение (4.5).

Определение 4.4. Симметрическая матрица $G$ называется допустимой, если она удовлетворяет матричному квадратному уравнению (4.5). Решение $\Gamma=$ $G(1-U G)^{-1}$ называется допустимым, если матрица параметров $G$ допустима.

Матричное квадратное уравнение (4.5) для симметрической матрицы $G$ является частным случаем так называемого алгебраического уравнения Риккати (см. например, [9]). Класс таких уравнений инвариантен относительно дробнолинейных преобразований, как это вытекает из следующей леммы:

Лемма 4.5. Если симметрическая матрица G удовлетворяет матричному квадратному уравнению

$$
G R G+Q G+G Q+P=0
$$

с некоторыми диагональными матрицами $P, Q, R$, то эквивалентная матрица $\widetilde{G}=(C+D G)(A+B G)^{-1}$ будет удовлетворять тому жсе уравнению:

$$
\widetilde{G} \widetilde{R} \widetilde{G}+\widetilde{Q} \widetilde{G}+\widetilde{G} \widetilde{Q}+\widetilde{P}=0,
$$

$2 \partial e$

$$
\begin{aligned}
& \widetilde{P}=D^{2} P-2 C D Q+C^{2} R, \\
& \widetilde{Q}=-B D P+(A D+B C) Q-A C R, \\
& \widetilde{R}=B^{2} P-2 A B Q+A^{2} R .
\end{aligned}
$$

Доказательство получается прямым вычислением с помощью тождества (3.7).

Следствие 4.6. Класс допустимъх решений основной системы (3.1) инвариантен относительно $\left[S L_{2}\right]^{n}$-действия (3.5).

Элементы $\Delta_{1}, \ldots, \Delta_{n}$ диагональной матрицы

$$
\Delta=Q^{2}-P R
$$

являются инвариантами $\left[S L_{2}\right]^{n}$-действия (4.8).

Следующий шаг - это параметризация слабо нелинейных решений уравнений ассоциативности WDVV решениями алгебраического уравнения Риккати (4.5) с предписанными коэффициентами, удовлетворяющими условию

$$
\left|p_{i}\right|^{2}+\left|q_{i}\right|^{2}+\left|r_{i}\right|^{2} \neq 0, \quad i=1, \ldots, n .
$$

Сначала упростим матричное квадратное уравнение с помощью преобразований (4.8).

Лемма 4.7. 1) Для допустимой неприводимой матрицъ G матричное квадратное уравнение (4.5) эквивалентно по модулю преобразования (4.8) уравнению

$$
G^{2}=\Delta,
$$


где матрица $\Delta$ задана формулой (4.9).

2) Для допустимой неприводимой матрицъ $G$ матрица $\Delta$ пропорииональна единичной матрице,

$$
\Delta_{1}=\cdots=\Delta_{n}=: \rho^{2} .
$$

Доказательство. Если все элементы матрицы $R$ отличны от нуля, то уравнение (4.5) может быть приведено к канонической форме (4.10) преобразовани$\mathrm{em}$

$$
G \mapsto A G A+B
$$

с подходящими диагональными матрицами $A$ и $B$. Это частный случай преобразования (4.8). Если $r_{i}=0$ для некоторого $i$, то можно предположить, что $p_{i} \neq 0$. Применим дробно-линейное преобразование (3.6) при $A=1-E_{i}$, $B=-E_{i}, C=E_{i}, D=1-E_{i}$, т. е. $G \mapsto \widetilde{G}=\left[G+E_{i}(1-G)\right]\left[1-E_{i}(1+G)\right]^{-1}$, где матрица $E_{i}$ имеет вид (2.2). Такое преобразование применимо, только если матрица $1-E_{i}(1+G)$ невырожденна. Легко видеть, что детерминант этой матрицы равен $\pm g_{i i}=\gamma_{i i}(0)$. Если $g_{i i}=0$, но решение неприводимо, то можно сделать сдвиг $\mathbf{u} \mapsto \mathbf{u}+\mathbf{u}^{0}$, чтобы получить матрицу $G^{\prime}=\Gamma\left(\mathbf{u}^{0}\right)$, где $g_{i i}^{\prime} \neq 0$. После преобразования получаем $\tilde{r}_{i}=p_{i} \neq 0$.

Для того чтобы доказать вторую часть леммы 4.7, достаточно заметить, что любой собственный вектор $f$ матрицы $G$ с собственным значением $\lambda$ также является собственным вектором матрицы $G^{2}$ с собственным значением $\lambda^{2}$. Итак, если $e_{i}$ и $e_{j}$ являются $i$-м и $j$-м базисными векторами и $\Delta_{i} \neq \Delta_{j}$, то эти векторы принадлежат соответственно суммам корневых подпространств $R\left(\sqrt{\Delta_{i}}\right) \oplus R\left(-\sqrt{\Delta_{i}}\right)$ и $R\left(\sqrt{\Delta_{j}}\right) \oplus R\left(-\sqrt{\Delta_{j}}\right)$ матрицы $G$. Так как эти корневые подпространства симметрических матриц ортогональны, матрица $G$ должна иметь блок-диагональную форму в этом же базисе.

Основная теорема 1.2 прямо следует из приведенного выше анализа.

Напомним что реконструкция решения уравнений ассоциативности с данными коэффициентами вращения (1.14) зависит от выбора решения линейной системы уравнений (1.6), (1.7). Мы теперь применим эту процедуру для того, чтобы построить слабо нелинейные решения уравнений WDVV. Удобно отдельно рассмотреть случаи $\rho \neq 0$ и $\rho=0$.

Случай 1. Собственные значения симметрической матрицы $G$, удовлетворяющей условию $G^{2}=\rho^{2} \cdot 1$, равны $\pm \rho$. Пусть $k$ - число собственных значений, равных $-\rho$. Здесь мы будем рассматривать более подробно случай $k=1$. Удобнее иметь дело с матрицей $\widetilde{G}=G-\rho \cdot 1$, удовлетворяющей уравнению $\widetilde{G}^{2}+2 \rho \widetilde{G}=0$. Для случая $k=1$ эта матрица может быть представлена в виде

$$
\widetilde{G}=\left(\omega_{i} \omega_{j}\right), \quad \sum_{i=1}^{n} \omega_{i}^{2}=-2 \rho .
$$

Ей соответствует семейство решений вида (3.3). Подстановка $\tilde{u}_{k}=-\log \left[\omega_{k}^{2}\left(u_{k}-\right.\right.$ $\left.\left.u_{k}^{0}\right)\right], k=1, \ldots, n$, с произвольными константами $u_{k}^{0}$, удовлетворяющими условию $\sum_{k=1}^{n} u_{k}^{0}=0$, дает следующие коэффициенты вращения, удовлетворяющие уравнениям Дарбу-Егорова:

$$
\tilde{\gamma}_{i j}=\frac{e^{-\left(\tilde{u}_{i}+\tilde{u}_{j}\right) / 2}}{\sum_{k=1}^{n} e^{-\tilde{u}_{k}}}, \quad i \neq j .
$$


В дальнейшем мы опустим все «тильды». Система (1.6)-(1.7) может быть легко решена:

$$
\psi_{i i}=\frac{2 e^{-u_{i}}}{D}-1, \quad \psi_{i j}=\frac{2 e^{-\left(u_{i}+u_{j}\right) / 2}}{D}, \quad i \neq j, \quad \text { где } D=\sum_{k=1}^{n} e^{-u_{k}} .
$$

Вычисление квадратур (1.9) дает следующие выражения для матрицы $\Omega$ вторых производных потенциала (см. (1.8)):

$$
\Omega_{i j}=u_{i} \delta_{i j}+\frac{4 e^{-\left(u_{i}+u_{j}\right) / 2}}{D} .
$$

Плоские координаты задаются линейными комбинациями столбцов этой матрицы. Выбор первого столбца дает егоровскую метрику

$$
d s^{2}=\left(1-4 \frac{e^{-u_{1}}}{D}\right) d u_{1}^{2}+4 \sum_{i=1}^{n} \frac{e^{-u_{1}-u_{i}}}{D^{2}} d u_{i}^{2}
$$

с плоскими координатами

$$
v_{1}=u_{1}+\frac{4 e^{-u_{1}}}{D}, \quad v_{i}=\frac{4 e^{-\left(u_{1}+u_{i}\right) / 2}}{D} \quad \text { при } i \neq 1 .
$$

Решая эти уравнения относительно канонических координат $u_{i}$, получаем

$$
u_{1}=v_{1}-\sqrt{4-\sigma}-2, \quad u_{i}=v_{1}-\sqrt{4-\sigma}-2+2 \log \frac{2+\sqrt{4-\sigma}}{v_{i}} \quad \text { при } i \neq 1,
$$

где $\sigma=\sum_{k=2}^{n} v_{k}^{2}$. Интегрируя квадратуры (4.11), выводим следующее выражение для потенциала, являющегося слабо нелинейным решением уравнений ассоциативности WDVV:

$$
F=\frac{1}{6} v_{1}^{3}+\frac{1}{2} v_{1} \sigma-\sum_{k=2}^{n} v_{k}^{2} \log v_{k}-\frac{1}{3}(2+\sigma) \sqrt{4-\sigma}+\sigma \log (2+\sqrt{4-\sigma}) .
$$

Также можно получить явное описание интегрируемой иерархии, ассоциированной в смысле [4] с (4.12). Напомним, что иерархия состоит из бесконечного семейства коммутирующих потоков, помеченных парой индексов $(\alpha, p)$, $\alpha=1, \ldots, n, p=0,1,2, \ldots$ Эти потоки имеют вид

$$
\frac{\partial v^{\gamma}}{\partial t^{\alpha, p}}=\partial_{x}\left(\nabla^{\gamma} \theta_{\alpha, p+1}(v)\right)
$$

Производящие функции

$$
\theta_{\alpha}(v, z)=\sum_{p=0}^{\infty} \theta_{\alpha, p}(v) z^{p}
$$

величин $\theta_{\alpha, p}(v)$ (деформированные плоские координаты) могут быть найдены в квадратурах,

$$
d \theta_{\alpha}(v, z)=\sum_{i=1}^{n} h_{i} \Psi_{i \alpha} d u_{i}, \quad \alpha=1, \ldots, n,
$$


из базиса $\Psi_{i \alpha}(v, z), \alpha=1, \ldots, n$, «волновых функций», определенных из системы

$$
\begin{aligned}
\frac{\partial \Psi_{i}}{\partial u_{j}} & =\gamma_{i j} \Psi_{j}, \quad i \neq j \\
\sum_{k=1}^{n} \frac{\partial \Psi_{i}}{\partial u_{k}} & =z \Psi_{i}
\end{aligned}
$$

Базис $\Psi_{i \alpha}$ может быть удобным образом отнормирован условиями

$$
\sum_{\alpha=1}^{n} \Psi_{i \alpha}(v,-z) \Psi_{j \alpha}(v, z)=\delta_{i j}
$$

В нашем случае нормированные волновые функции имеют вид

$$
\Psi_{i \alpha}=\frac{2 e^{z u_{\alpha}}}{\sqrt{1-4 z^{2}}}\left[\left(z-\frac{1}{2}\right) \delta_{i \alpha}+\frac{e^{-\frac{u_{i}+u_{\alpha}}{2}}}{D}\right]
$$

Это дает

$\theta_{\alpha}=\frac{1}{\sqrt{1-4 z^{2}}}\left\{\left[\frac{1}{z}\left(e^{z u_{1}}-1\right)-e^{z u_{1}}\left(u_{1}+2\right)+2\right] \delta_{\alpha 1}+v_{\alpha} e^{z u_{\alpha}}\right\}, \quad \alpha=1, \ldots, n$.

Случай 2. Теперь мы рассмотрим второй тип решений, параметризованных симметрическими матрицами $G$, удовлетворяющими условию $G^{2}=0$. В этом случае получаем решение уравнений ассоциативности WDVV, также удовлетворяющее условию квазиоднородности.

Все собственные значения матрицы $G$ равны 0. Все жордановы блоки имеют порядки 1 или 2. Рассмотрим простейший случай только одного блока порядка 2. Элементы матрицы $G=\left(g_{i j}\right)$ можно записать в форме

$$
g_{i j}=\omega_{i} \omega_{j}, \quad \sum_{i=1}^{n} \omega_{i}^{2}=0
$$

Соответствующее решение уравнений ассоциативности WDVV может быть получено из тривиального (т. е. кубического)

$$
F(v)=\frac{1}{6} \sum_{i, j, k} c_{i j k} v^{i} v^{j} v^{k}
$$

преобразованием инверсии из [5] (см. дополнение В и предложение 3.14). Здесь $c_{i j k}$ - структурные константы полупростой фробениусовой алгебры

$$
\mathscr{A}=\operatorname{span}\left(e_{1}, \ldots, e_{n}\right), \quad\left\langle e_{i} \cdot e_{j}, e_{k}\right\rangle=c_{i j k}, \quad\left\langle e_{i}, e_{j}\right\rangle=\delta_{i+j, n+1},
$$

с единицей $e_{1}$ и тривиальной градуировкой $\operatorname{deg} e_{i}=0$ для всех $i$. Напоминаем, что структурные константы могут быть представлены в форме

$$
c_{i j k}=\sum_{s=1}^{n} \frac{a_{s i} a_{s j} a_{s k}}{a_{s 1}},
$$


где матрица $\left(a_{i j}\right)$ удовлетворяет условию

$$
\sum_{s=1}^{n} a_{s i} a_{s j}=\delta_{i+j, n+1}
$$

Для нашей конструкции можно выбрать матрицу таким образом, что

$$
a_{i 1}=\omega_{i}, \quad i=1, \ldots, n \text {. }
$$

После подстановки

$$
\begin{aligned}
\hat{v}^{1} & =\frac{1}{2} \frac{v_{\alpha} v^{\alpha}}{v^{n}}, \\
\hat{v}^{\alpha} & =\frac{v^{\alpha}}{v^{n}}, \quad \alpha \neq 1, n, \\
\hat{v}^{n} & =-\frac{1}{v^{n}}
\end{aligned}
$$

получается искомое решение $\widehat{F}$ уравнений ассоциативности WDVV в форме

$$
\widehat{F}(\hat{v})=\frac{1}{2} \hat{v}^{1} \hat{v}_{\alpha} \hat{v}^{\alpha}+\left(\hat{v}^{n}\right)^{2} F(v)=\frac{1}{2}\left(\hat{v}^{1}\right)^{2} \hat{v}^{n}+\frac{1}{2} \sum_{\alpha=2}^{n-1} \hat{v}^{1} \hat{v}^{\alpha} \hat{v}^{n-\alpha+1}+\frac{P\left(\hat{v}^{2}, \ldots, \hat{v}^{n-1}\right)}{\hat{v}^{n}} .
$$

Здесь ${ }^{1)} P\left(\hat{v}^{2}, \ldots, \hat{v}^{n-1}\right)$ - некоторый полином четвертой степени. Потенциал $\widehat{F}$ удовлетворяет условию квазиоднородности

$$
\widehat{E} \widehat{F}=\widehat{F}, \quad \widehat{E}=\hat{v}^{1} \frac{\partial}{\partial \hat{v}^{1}}-\hat{v}^{n} \frac{\partial}{\partial \hat{v}^{n}} .
$$

\section{ЛиТЕРАТУРА}

[1] G. Darboux, Leçons sur systèmes orthogonaux et les coordonnées curvilignes, Paris, 1910.

[2] Б. А. Дубровин, Вполне интегрируемые гамильтоновы системь, связанные с матричными операторами, и абелевы многообразия, Функц. анализ и его прил., 11:4 (1977), 28-41.

[3] Б. А. Дубровин, K дифференииалъной геометрии сильно интегрируемъх систем гидродинамического типа, Функц. анализ и его прил., 24:4 (1990), 25-30.

[4] B. Dubrovin, Integrable systems in topological field theory, Nucl. Phys. B, 379:3 (1992), 627-689.

[5] B. Dubrovin, Geometry of $2 D$ topological field theories, in: Integrable Systems and Quantum Groups, Montecatini, Terme, 1993, Lecture Notes in Math., vol. 1620, Springer-Verlag, Berlin, 1996, 120-348.

[6] Б. А. Дубровин, С. П. Новиков, Гамильтонов формализм одномерных систем гидродинамического типа и метод усреднения Боголюбова-Уизема, Докл. АН CCCP, 270 (1983), 781-785.

[7] Д. Ф. Егоров, Об одном классе ортогональных систем, Ученые записки Московского университета, Секц. физ.-мат., 18 (1901), 1-239.

[8] G. A. El, A. M. Kamchatnov, M. V. Pavlov, S. A. Zykov, Kinetic equation for a soliton gas and its hydrodynamic reductions, J. Nonlinear Sci., 21:2 (2011), 151-191.

1)Этот пример также был рассмотрен в [10] в ином контексте. Наша формула (4.13) отличается от найденной в работе [10]. 
[9] P. Lancaster, L. Rodman, Algebraic Riccati Equations, Clarendon Press, Oxford University Press, Oxford, 1995.

[10] J. W. van de Leur, R. Martini, The construction of Frobenius manifolds from KP tau-functions, Comm. Math. Phys., 205:3 (1999), 587-616.

[11] С. П. Царев, Геометрия гамильтоновых систем гидродинамического типа. Обобщенный метод годографа, Изв. АН СССР, сер. матем., 54:5 (1991), 1048-1068.

SISSA, Триест

Московский государственный университет

Поступило в редакцию

им. М. В. Ломоносова,

лаборатория геометрических методов математической физики

Математический институт им. В. А. Стеклова

e-mail: dubrovin@sissa.it

Университет Саленто, Лечче,

факультет математических, физических и естественных наук

Институт физики металлов Уральского отделения РАН

e-mail: serg@imp.uran.ru

Московский государственный университет им. М. В. Ломоносова, лаборатория геометрических методов математической физики

Физический институт им. П. Н. Лебедева РАН,

сектор математической физики

e-mail: maxim.pavlov@mtu-net.ru,maxim@math.sinica.edu.tw 